\title{
Generalized commutative quaternions of the Fibonacci type
}

\section{Anetta Szynal-Liana ${ }^{1}$ (D) Iwona Włoch ${ }^{1}$ (D)}

Received: 28 March 2021 / Accepted: 6 September 2021 / Published online: 17 November 2021

(C) The Author(s) 2021

\begin{abstract}
Quaternions are a four-dimensional hypercomplex number system discovered by Hamilton in 1843 and next intensively applied in mathematics, modern physics, computer graphics and other fields. After the discovery of quaternions, modified quaternions were also defined in such a way that commutative property in multiplication is possible. That number system called as commutative quaternions is intensively studied and used for example in signal processing. In this paper we define generalized commutative quaternions and next based on them we define and explore Fibonacci type generalized commutative quaternions.
\end{abstract}

Keywords Quaternions · Generalized quaternions · Fibonacci numbers · Horadam numbers

Mathematics Subject Classification 11B37 · 11 B39

\section{Introduction}

One of the problems in applying quaternions is their noncommutative structure. In other words, a different order of multiplication of quaternions results in different quaternions. That structure makes hard to conduct applications among other to engineering problems, see for example [22]. Modified quaternions were proposed by Serge in [26], so that commutative property in multiplication is possible. Quaternions and commutative quaternions belong to the class of hypercomplex

Anetta Szynal-Liana

aszynal@prz.edu.pl

Iwona Włoch

iwloch@prz.edu.pl

1 Rzeszow University of Technology, Faculty of Mathematics and Applied Physics, Al.

Powstańców Warszawy 12, Rzeszów 35-959, Poland 
numbers. Let $N \geq 0$ be an integer. Hypercomplex numbers are defined by the expression (see $[5,21,27]$ )

$$
\mathbf{x}=\sum_{i=0}^{N-1} x_{i} e_{i}
$$

where $x_{i} \in \mathbb{R}$ are called components and $e_{i}$ are called units. The element $e_{0}$ is the scalar or the real element and it may be identified with the real number 1 . For $i=1, \ldots, N-1$ we have $e_{i} \notin \mathbb{R}$.

Let $\mathbf{x}=\sum_{i=0}^{N-1} x_{i} e_{i}$ and $\mathbf{y}=\sum_{i=0}^{N-1} y_{i} e_{i}$ be any two hypercomplex numbers. We define equality, addition and multiplication by scalar in the following way:

$$
\begin{gathered}
\mathbf{x}=\mathbf{y} \text { only if } x_{i}=y_{i}, i=0,1, \ldots, N-1 \text { (equality) } \\
\mathbf{x}+\mathbf{y}=\sum_{i=0}^{N-1}\left(x_{i}+y_{i}\right) e_{i} \quad \text { (addition) } \\
\left.s \cdot \mathbf{x}=\sum_{i=0}^{N-1}\left(s \cdot x_{i}\right) e_{i} \quad \text { (multiplication by scalar } s \in \mathbb{R}\right) .
\end{gathered}
$$

The zero element is the element with all components equal to zero. From these definitions it follows that the addition satisfies commutative and associative properties and the zero element exists, see [6].

Important in defining the multiplication of hypercomplex numbers is that the product of hypercomplex numbers is still a hypercomplex number.

For real and complex numbers the product operation satisfies the following properties: distributive with respect to the sum, associative, commutative, does not have divisors of zero. It can be shown (see [25]) that, only the real and complex numbers can satisfy these four properties all together. As far as other systems are concerned, if they satisfy the first two properties they can satisfy just one of the last two.

Analogously as for quaternions the class (family) of hypercomplex numbers can be partitioned into two subclasses (subfamilies) non-commutative hypercomplex system and commutative hypercomplex system.

A generalized noncommutative quaternion $\mathbf{x}$ is a vector in 4-dimensional vectorspace of the form $\mathbf{x}=x_{0}+x_{1} e_{1}+x_{2} e_{2}+x_{3} e_{3}$, where quaternionic units $e_{1}, e_{2}, e_{3}$ satisfy the equalities

$$
\begin{gathered}
e_{1}^{2}=-\alpha, \quad e_{2}^{2}=-\beta, \quad e_{3}^{2}=-\alpha \beta, \\
e_{1} e_{2}=-e_{2} e_{1}=e_{3}, \quad e_{2} e_{3}=-e_{3} e_{2}=\beta e_{1} \text { and } e_{3} e_{1}=-e_{1} e_{3}=\alpha e_{2},
\end{gathered}
$$

where $\alpha, \beta \in \mathbb{R}$. The class of all generalized noncommutative quaternions we denote by $\mathbb{Q}_{\alpha \beta}^{n}$. For special values of $\alpha$ and $\beta$ we obtain well-known subclasses of noncommutative quaternions

$\alpha=\beta=1-$ real quaternions,

$\alpha=1, \beta=-1-$ split quaternions,

$\alpha=1, \beta=0$ - semi-quaternions, 
$\alpha=-1, \beta=0-$ split semiquaternions,

$\alpha=\beta=0-1 / 4$ quaternions.

Algebraic properties of generalized quaternions were described in [16]. Motivated by above we introduce generalized commutative quaternions as follows.

A generalized commutative quaternion $\mathbf{x}$ is a vector of the form $\mathbf{x}=x_{0}+x_{1} e_{1}+x_{2} e_{2}+x_{3} e_{3}$, where quaternionic units $e_{1}, e_{2}, e_{3}$ satisfy the equalities

$$
\begin{gathered}
e_{1}^{2}=\alpha, \quad e_{2}^{2}=\beta, \quad e_{3}^{2}=\alpha \beta, \\
e_{1} e_{2}=e_{2} e_{1}=e_{3}, \quad e_{2} e_{3}=e_{3} e_{2}=\beta e_{1} \text { and } e_{3} e_{1}=e_{1} e_{3}=\alpha e_{2},
\end{gathered}
$$

where $\alpha, \beta \in \mathbb{R}$. The family of all generalized commutative quaternions we denote by $\mathbb{T}_{\alpha \beta}^{c}$. For special $\alpha$ and $\beta$ there are the following subclasses of commutative quaternions

$\alpha<0, \beta=1$ - elliptic quaternions, in particular canonical (Segre's) quaternions if $\alpha=-1$,

$\alpha=0, \beta=1-$ parabolic quaternions,

$\alpha>0, \beta=1-$ hyperbolic quaternions, in particular canonical or bihyperbolic numbers if $\alpha=1$,

$\alpha=-1, \beta=1-$ tessarines or complex hyperbolic numbers,

$\alpha=-1, \beta=-1-$ bicomplex numbers,

$\alpha=1, \beta=-1-$ hyperbolic complex numbers.

Note that the above special cases are well-known and studied in the literature. It suffices to mention that in [6] Catoni et al. studied algebraic properties of three types of commutative quaternions i.e. elliptic, parabolic and hyperbolic quaternions. Although the theory of commutative quaternions is becoming important in recent years and has interesting applications in distinct areas of mathematics and modern physics, the study of commutative quaternions is focus mainly on elliptic quaternions, see for example [17-20]. Tessarines were introduced by Cocle in 1848 and next that theory was developed in the sequences of articles [7-10]. Bicomplex numbers were discovered by Serge, see [26] and recently studied by Rochon in [24]. Hyperbolic complex numbers also named as complex numbers with hyperbolic coefficients were investigated in [1]. Bihyperbolic numbers are one of the extension of hyperbolic numbers, see [4, 23].

In recent years the theory of hypercomplex numbers is complemented by their connections with Fibonacci sequences, for more details see the book [27].

Generalized noncommutative Fibonacci quaternions were introduced by Horadam in [15]. In [2, 11-13] the authors presented some properties of the generalized noncommutative Fibonacci quaternions.

In this paper we define generalized commutative quaternions of the Fibonacci type. 


\section{Generalized commutative quaternions of the Fibonacci type}

Let $n \geq 0$ be an integer. The $n$th Fibonacci number $F_{n}$ is a number defined recursively by the second-order linear recurrence relation $F_{n}=F_{n-1}+F_{n-2}$, for $n \geq 2$ with $F_{0}=0, F_{1}=1$.

The Fibonacci numbers are generalized in different ways, see for example the list in [3] and [27]. Consider the well-known generalization of Fibonacci numbers given by Horadam in [14].

Let $p, q, n$ be integers. For $n \geq 0$ Horadam defined the numbers $W_{n}=$ $W_{n}\left(W_{0}, W_{1} ; p, q\right)$ by the recursive equation

$$
W_{n}=p \cdot W_{n-1}-q \cdot W_{n-2},
$$

for $n \geq 2$ with fixed real numbers $W_{0}, W_{1}$.

For the historical reasons these numbers were later called the Horadam numbers.

Let $t_{1}, t_{2}$ be the two distinct real roots of the equation

$$
t^{2}-p t+q=0 \text {. }
$$

Then, the Binet type formula for the Horadam numbers has the form

$$
W_{n}=A t_{1}^{n}+B t_{2}^{n}
$$

where

$$
\begin{aligned}
& t_{1}=\frac{p-\sqrt{p^{2}-4 q}}{2}, \\
& t_{2}=\frac{p+\sqrt{p^{2}-4 q}}{2}, \\
& A=\frac{W_{1}-W_{0} t_{2}}{t_{1}-t_{2}}, \\
& B=\frac{W_{0} t_{1}-W_{1}}{t_{1}-t_{2}} .
\end{aligned}
$$

For special $W_{0}, W_{1}, p, q$ the equation (3) defines selected numbers of the Fibonacci type:

the Fibonacci numbers $F_{n}=W_{n}(0,1 ; 1,-1)$, the Lucas numbers $L_{n}=W_{n}(2,1 ; 1,-1)$, the Pell numbers $P_{n}=W_{n}(0,1 ; 2,-1)$, the Pell-Lucas numbers $Q_{n}=W_{n}(2,2 ; 2,-1)$, the Jacobsthal numbers $J_{n}=W_{n}(0,1 ; 1,-2)$, the Jacobsthal-Lucas numbers $j_{n}=W_{n}(2,1 ; 1,-2)$, the balancing numbers $B_{n}=W_{n}(0,1 ; 6,1)$, the Lucas-balancing numbers $C_{n}=W_{n}(1,3 ; 6,1)$ etc.

Let $n \geq 0$ be an integer. The $n$th generalized commutative Horadam quaternion gc $\mathscr{H}_{n}$ is defined as

$$
g c \mathscr{H}_{n}=W_{n}+W_{n+1} e_{1}+W_{n+2} e_{2}+W_{n+3} e_{3},
$$

where $W_{n}$ is the $n$th Horadam number and $e_{1}, e_{2}, e_{3}$ are units satisfy (1) and (2). 
Theorem 1 (Binet type formula for generalized commutative Horadam quaternions) Let $n \geq 0$ be an integer. Then

$$
g c \mathscr{H}_{n}=A t_{1}^{n}\left(1+t_{1} e_{1}+t_{1}^{2} e_{2}+t_{1}^{3} e_{3}\right)+B t_{2}^{n}\left(1+t_{2} e_{1}+t_{2}^{2} e_{2}+t_{2}^{3} e_{3}\right),
$$

where $t_{1}, t_{2}, A$ and $B$ are given by (5).

Proof Using (6) and (4) we have

$$
\begin{aligned}
g c \mathscr{H}_{n}= & W_{n}+W_{n+1} e_{1}+W_{n+2} e_{2}+W_{n+3} e_{3} \\
= & A t_{1}^{n}+B t_{2}^{n}+\left(A t_{1}^{n+1}+B t_{2}^{n+1}\right) e_{1}+\left(A t_{1}^{n+2}+B t_{2}^{n+2}\right) e_{2} \\
& +\left(A t_{1}^{n+3}+B t_{2}^{n+3}\right) e_{3} \\
= & A t_{1}^{n}\left(1+t_{1} e_{1}+t_{1}^{2} e_{2}+t_{1}^{3} e_{3}\right)+B t_{2}^{n}\left(1+t_{2} e_{1}+t_{2}^{2} e_{2}+t_{2}^{3} e_{3}\right)
\end{aligned}
$$

and the result follows.

For simplicity of notation let

$$
\begin{aligned}
& \hat{t_{1}}=1+t_{1} e_{1}+t_{1}^{2} e_{2}+t_{1}^{3} e_{3}, \\
& \hat{t_{2}}=1+t_{2} e_{1}+t_{2}^{2} e_{2}+t_{2}^{3} e_{3} .
\end{aligned}
$$

Then we can write (7) as

$$
g c \mathscr{H}_{n}=A t_{1}^{n} \hat{t_{1}}+B t_{2}^{n} \hat{t_{2}} \text {. }
$$

Theorem 2 (Catalan type identity for generalized commutative Horadam quaternions) Let $n \geq 0, r \geq 0$ be integers such that $n \geq r$. Then

$$
\begin{aligned}
& g c \mathscr{H}_{n-r} \cdot g c \mathscr{H}_{n+r}-\left(g c \mathscr{H}_{n}\right)^{2} \\
& =A B t_{1}^{n} t_{2}^{n}\left(\left(\frac{t_{1}}{t_{2}}\right)^{r}+\left(\frac{t_{2}}{t_{1}}\right)^{r}-2\right) \hat{t_{1}} \hat{t_{2}},
\end{aligned}
$$

where $t_{1}, t_{2}, A, B$ and $\hat{t_{1}}, \hat{t_{2}}$ are given by (5) and (8), respectively.

Proof By formula (7) we have

$$
\begin{aligned}
g c \mathscr{H}_{n+r} \cdot g c \mathscr{H}_{n-r}-\left(g c \mathscr{H}_{n}\right)^{2} \\
=\left(A t_{1}^{n+r} \hat{t_{1}}+B t_{2}^{n+r} \hat{t_{2}}\right) \cdot\left(A t_{1}^{n-r} \hat{t_{1}}+B t_{2}^{n-r} \hat{t_{2}}\right)-\left(A t_{1}^{n} \hat{t_{1}}+B t_{2}^{n} \hat{t_{2}}\right)^{2} \\
=A t_{1}^{n+r} \hat{t_{1}} A t_{1}^{n-r} \hat{t_{1}}+A t_{1}^{n+r} \hat{t_{1}} B t_{2}^{n-r} \hat{t_{2}}+B t_{2}^{n+r} \hat{t_{2}} A t_{1}^{n-r} \hat{t_{1}}+B t_{2}^{n+r} \hat{t_{2}} B t_{2}^{n-r} \hat{t_{2}} \\
-A t_{1}^{n} \hat{t_{1}} A t_{1}^{n} \hat{t_{1}}-A t_{1}^{n} \hat{t_{1}} B t_{2}^{n} \hat{t_{2}}-B t_{2}^{n} \hat{t_{2}} A t_{1}^{n} \hat{t_{1}}-B t_{2}^{n} \hat{t_{2}} B t_{2}^{n} \hat{t_{2}} \\
=A t_{1}^{n+r} \hat{t_{1}} B t_{2}^{n-r} \hat{t_{2}}+B t_{2}^{n+r} \hat{t_{2}} A t_{1}^{n-r} \hat{t_{1}}-A t_{1}^{n} \hat{t_{1}} B t_{2}^{n} \hat{t_{2}}-B t_{2}^{n} \hat{t_{2}} A t_{1}^{n} \hat{t_{1}} \\
=A B t_{1}^{n} t_{2}^{n}\left(\left(\frac{t_{1}}{t_{2}}\right)^{r}+\left(\frac{t_{2}}{t_{1}}\right)^{r}-2\right) \hat{t_{1}} \hat{t_{2}},
\end{aligned}
$$

which ends the proof. 
Note that for $r=1$ we get the Cassini type identity for generalized commutative Horadam quaternions.

Corollary 3 (Cassini type identity for generalized commutative Horadam quaternions) Let $n \geq 1$ be an integer. Then

$$
\begin{gathered}
g c \mathscr{H}_{n+1} \cdot g c \mathscr{H}_{n-1}-\left(g c \mathscr{H}_{n}\right)^{2} \\
=A B t_{1}^{n} t_{2}^{n}\left(\frac{t_{1}}{t_{2}}+\frac{t_{2}}{t_{1}}-2\right) \hat{t_{1}} \hat{t_{2}},
\end{gathered}
$$

where $t_{1}, t_{2}, A, B$ and $\hat{t_{1}}, \hat{t_{2}}$ are given by (5) and (8), respectively.

Theorem 4 (d'Ocagne type identity for generalized commutative Horadam quaternions) Let $m \geq 0, n \geq 0$ be integers such that $m \geq n$. Then

$$
\begin{aligned}
& g c \mathscr{H}_{m} \cdot g c \mathscr{H}_{n+1}-g c \mathscr{H}_{m+1} \cdot g c \mathscr{H}_{n} \\
& =A B t_{1}^{n} t_{2}^{n}\left(t_{1}-t_{2}\right)\left(t_{2}^{m-n}-t_{1}^{m-n}\right) \hat{t_{1}} \hat{t_{2}},
\end{aligned}
$$

where $t_{1}, t_{2}, A, B$ and $\hat{t_{1}}, \hat{t_{2}}$ are given by (5) and (8), respectively.

Proof By formula (7) we have

$$
\begin{aligned}
& g c \mathscr{H}_{m} \cdot g c \mathscr{H}_{n+1}-g c \mathscr{H}_{m+1} \cdot g c \mathscr{H}_{n} \\
= & \left(A t_{1}^{m} \hat{t_{1}}+B t_{2}^{m} \hat{t_{2}}\right)\left(A t_{1}^{n+1} \hat{t_{1}}+B t_{2}^{n+1} \hat{t_{2}}\right) \\
& -\left(A t_{1}^{m+1} \hat{t_{1}}+B t_{2}^{m+1} \hat{t_{2}}\right)\left(A t_{1}^{n} \hat{t_{1}}+B t_{2}^{n} \hat{t_{2}}\right) \\
= & A t_{1}^{m} \hat{t_{1}} A t_{1}^{n+1} \hat{t_{1}}+B t_{2}^{m} \hat{t_{2}} A t_{1}^{n+1} \hat{t_{1}}+A t_{1}^{m} \hat{t_{1} B t_{2}^{n+1}} \hat{t_{2}}+B t_{2}^{m} \hat{t_{2}} B t_{2}^{n+1} \hat{t_{2}} \\
& -A t_{1}^{m+1} \hat{t_{1}} A t_{1}^{n} \hat{t_{1}}-B t_{2}^{m+1} \hat{t_{2}} A t_{1}^{n} \hat{t_{1}}-A t_{1}^{m+1} \hat{t_{1}} B t_{2}^{n} \hat{t_{2}}-B t_{2}^{m+1} \hat{t_{2}} B t_{2}^{n} \hat{t_{2}} \\
= & B t_{2}^{m} \hat{t_{2}} A t_{1}^{n+1} \hat{t_{1}}+A t_{1}^{m} \hat{t_{1}} B t_{2}^{n+1} \hat{t_{2}}-B t_{2}^{m+1} \hat{t_{2}} A t_{1}^{n} \hat{t_{1}}-A t_{1}^{m+1} \hat{t_{1} B t_{2}^{n} \hat{t_{2}}} \\
= & A B t_{1}^{n} t_{2}^{n}\left(t_{1} t_{2}^{m-n}+t_{1}^{m-n} t_{2}-t_{2} t_{2}^{m-n}-t_{1} t_{1}^{m-n}\right) \hat{t_{1}} \hat{t_{2}} \\
= & A B t_{1}^{n} t_{2}^{n}\left(t_{2}^{m-n}\left(t_{1}-t_{2}\right)-t_{1}^{m-n}\left(t_{1}-t_{2}\right)\right) \hat{t_{1}} \hat{t_{2}} \\
= & A B t_{1}^{n} t_{2}^{n}\left(t_{1}-t_{2}\right)\left(t_{2}^{m-n}-t_{1}^{m-n}\right) \hat{t_{1}} \hat{t_{2}},
\end{aligned}
$$

which ends the proof.

Moreover, by simple calculations we obtain

$$
\begin{gathered}
t_{1}+t_{2}=p, \quad t_{1} t_{2}=q, \\
t_{1}^{2}+t_{2}^{2}=\left(t_{1}+t_{2}\right)^{2}-2 t_{1} t_{2}=p^{2}-2 q, \\
t_{1}^{3}+t_{2}^{3}=\left(t_{1}+t_{2}\right)^{3}-3 t_{1} t_{2}\left(t_{1}+t_{2}\right)=p^{3}-3 p q
\end{gathered}
$$

and 


$$
\begin{aligned}
& \hat{t_{1}} \hat{t_{2}}=\hat{t_{2}} \hat{t_{1}}=\left(1+t_{1} e_{1}+t_{1}^{2} e_{2}+t_{1}^{3} e_{3}\right)\left(1+t_{2} e_{1}+t_{2}^{2} e_{2}+t_{2}^{3} e_{3}\right) \\
& =1+t_{2} e_{1}+t_{2}^{2} e_{2}+t_{2}^{3} e_{3}+t_{1} e_{1}+t_{1} e_{1} t_{2} e_{1}+t_{1} e_{1} t_{2}^{2} e_{2}+t_{1} e_{1} t_{2}^{3} e_{3}+t_{1}^{2} e_{2} \\
& +t_{1}^{2} e_{2} t_{2} e_{1}+t_{1}^{2} e_{2} t_{2}^{2} e_{2}+t_{1}^{2} e_{2} t_{2}^{3} e_{3}+t_{1}^{3} e_{3}+t_{1}^{3} e_{3} t_{2} e_{1}+t_{1}^{3} e_{3} t_{2}^{2} e_{2}+t_{1}^{3} e_{3} t_{2}^{3} e_{3} \\
& =1+t_{2} e_{1}+t_{2}^{2} e_{2}+t_{2}^{3} e_{3}+t_{1} e_{1}+t_{1} t_{2} \alpha+t_{1} t_{2}^{2} e_{3}+t_{1} t_{2}^{3} \alpha e_{2}+t_{1}^{2} e_{2} \\
& +t_{1}^{2} t_{2} e_{3}+t_{1}^{2} t_{2}^{2} \beta+t_{1}^{2} t_{2}^{3} \beta e_{1}+t_{1}^{3} e_{3}+t_{1}^{3} t_{2} \alpha e_{2}+t_{1}^{3} t_{2}^{2} \beta e_{1}+t_{1}^{3} t_{2}^{3} \alpha \beta \\
& =1+t_{1} t_{2} \alpha+t_{1}^{2} t_{2}^{2} \beta+t_{1}^{3} t_{2}^{3} \alpha \beta+\left(t_{1}+t_{2}+t_{1}^{2} t_{2}^{3} \beta+t_{1}^{3} t_{2}^{2} \beta\right) e_{1} \\
& \quad+\left(t_{1}^{2}+t_{2}^{2}+t_{1} t_{2}^{3} \alpha+t_{1}^{3} t_{2} \alpha\right) e_{2}+\left(t_{1}^{3}+t_{2}^{3}+t_{1} t_{2}^{2}+t_{1}^{2} t_{2}\right) e_{3} \\
& =1+q \alpha+q^{2} \beta+q^{3} \alpha \beta+\left(p+p q^{2} \beta\right) e_{1}+\left(p^{2}-2 q\right)(1+q \alpha) e_{2}+\left(p^{3}-2 p q\right) e_{3} .
\end{aligned}
$$

Substituting the appropriate values of $p, q, W_{0}, W_{1}$ etc. and using the above calculations we can obtain Binet formulas, Catalan, Cassini and d'Ocagne identities for the mentioned numbers of the Fibonacci type.

Now we give matrix representation of commutative Horadam quaternions. Let $g c \mathscr{H}(n)=\left[\begin{array}{cc}g c \mathscr{H}_{n+1} & g c \mathscr{H}_{n} \\ g c \mathscr{H}_{n} & g c \mathscr{H}_{n-1}\end{array}\right]$ be a matrix with entries being generalized commutative Horadam quaternions.

Theorem 5 Let $n \geq 1$ be an integer. Then

$$
\left[\begin{array}{cc}
g c \mathscr{H}_{n+1} & g c \mathscr{H}_{n} \\
g c \mathscr{H}_{n} & g c \mathscr{H}_{n-1}
\end{array}\right]=\left[\begin{array}{ll}
g c \mathscr{H}_{2} & g c \mathscr{H}_{1} \\
g c \mathscr{H}_{1} & g c \mathscr{H}_{0}
\end{array}\right] \cdot\left[\begin{array}{cc}
p & 1 \\
-q & 0
\end{array}\right]^{n-1} .
$$

Proof If $n=1$, then by simple calculations the result immediately follows. Assume that the equality (9) is true for all integers $1,2, \ldots, n$. We shall prove that the equality is true for integer $n+1$. Using induction hypothesis we have

$$
\begin{aligned}
& {\left[\begin{array}{ll}
g c \mathscr{H}_{2} & g c \mathscr{H}_{1} \\
g c \mathscr{H}_{1} & g c \mathscr{H}_{0}
\end{array}\right] \cdot\left[\begin{array}{cc}
p & 1 \\
-q & 0
\end{array}\right]^{n-1} \cdot\left[\begin{array}{cc}
p & 1 \\
-q & 0
\end{array}\right]=\left[\begin{array}{cc}
g c \mathscr{H}_{n+1} & g c \mathscr{H}_{n} \\
g c \mathscr{H}_{n} & g c \mathscr{H}_{n-1}
\end{array}\right] \cdot\left[\begin{array}{cc}
p & 1 \\
-q & 0
\end{array}\right]} \\
& =\left[\begin{array}{ll}
p \cdot g c \mathscr{H}_{n+1}-q \cdot g c \mathscr{H}_{n} & g c \mathscr{H}_{n+1} \\
p \cdot g c \mathscr{H}_{n}-q \cdot g c \mathscr{H}_{n-1} & g c \mathscr{H}_{n}
\end{array}\right]=\left[\begin{array}{cc}
g c \mathscr{H}_{n+2} & g c \mathscr{H}_{n+1} \\
g c \mathscr{H}_{n+1} & g c \mathscr{H}_{n}
\end{array}\right],
\end{aligned}
$$

which ends the proof.

Properties of commutative Horadam quaternions allow the use determinant properties. Moreover

$$
\left[\begin{array}{ll}
g c \mathscr{H}_{2} & g c \mathscr{H}_{1} \\
g c \mathscr{H}_{1} & g c \mathscr{H}_{0}
\end{array}\right] \cdot\left[\begin{array}{cc}
p & 1 \\
-q & 0
\end{array}\right]=\left[\begin{array}{cc}
p & 1 \\
-q & 0
\end{array}\right]^{T} \cdot\left[\begin{array}{ll}
g c \mathscr{H}_{2} & g c \mathscr{H}_{1} \\
g c \mathscr{H}_{1} & g c \mathscr{H}_{0}
\end{array}\right]
$$

and 


$$
\operatorname{det}\left[\begin{array}{cc}
p & 1 \\
-q & 0
\end{array}\right]=\operatorname{det}\left[\begin{array}{cc}
p & 1 \\
-q & 0
\end{array}\right]^{T}=q .
$$

Consequently by algebraic operations and matrix algebra some interesting properties of commutative Horadam quaternions can be expected.

Acknowledgements The authors would like to thank the referees for helpful valuable suggestions which resulted in improvements to this paper.

\section{Declarations}

Conflict of interest The authors declare that they have no conflict of interest.

Open Access This article is licensed under a Creative Commons Attribution 4.0 International License, which permits use, sharing, adaptation, distribution and reproduction in any medium or format, as long as you give appropriate credit to the original author(s) and the source, provide a link to the Creative Commons licence, and indicate if changes were made. The images or other third party material in this article are included in the article's Creative Commons licence, unless indicated otherwise in a credit line to the material. If material is not included in the article's Creative Commons licence and your intended use is not permitted by statutory regulation or exceeds the permitted use, you will need to obtain permission directly from the copyright holder. To view a copy of this licence, visit http:// creativecommons.org/licenses/by/4.0/.

\section{References}

1. Akar, M., Yüce, S., Şahin, S.: On the dual hyperbolic numbers and the complex hyperbolic numbers. J. Comput. Sci. Comput. Math. 8(1), 1-6 (2018). https://doi.org/10.20967/jcscm.2018.01.001

2. Akyigit, M., Kosal, H.H., Tosun, M.: Fibonacci generalized quaternions. Adv. Appl. Clifford Algebr. 24, 631-641 (2014). https://doi.org/10.1007/s00006-014-0458-0

3. Bednarz, U., Włoch, I., Wołowiec-Musiał, M.: Total graph interpretation of the numbers of the Fibonacci type. J. Appl. Math. 2015, 7 (2015). https://doi.org/10.1155/2015/837917

4. Bilgin, M., Ersoy, S.: Algebraic properties of bihyperbolic numbers. Adv. Appl. Clifford Algebr. 30, 13 (2020). https://doi.org/10.1007/s00006-019-1036-2

5. Cartan, E.: Oeuvres Complètes. Gauthier Villars, Paris (1953)

6. Catoni, F., Boccaletti, D., Cannata, R., Catoni, V., Nichelatti, E., Zampetti, P.: The Mathematics of Minkowski Space-Time. Birkhäuser Verlag AG, Basel Boston Berlin (2008)

7. Cockle, J.: On a new imaginary in algebra. London Edinburgh Dublin Philos. Mag. J. Sci. 34, 37-47 (1849). https://doi.org/10.1080/14786444908646169

8. Cockle, J.: On certain functions resembling quaternions, and on a new imaginary in algebra. London Edinburgh Dublin Philos. Mag. J. Sci. 33, $435-439$ (1848). https://doi.org/10.1080/ 14786444808646139

9. Cockle, J.: On impossible equations, on impossible quantities, and on tessarines. London Edinburgh Dublin Philos. Mag. J. Sci. 37, 281-283 (1850). https://doi.org/10.1080/14786445008646598

10. Cockle, J.: On the symbols of algebra, and on the theory of tesarines. London Edinburgh Dublin Philos. Mag. J. Sci. 34, 406-410 (1849). https://doi.org/10.1080/14786444908646257

11. Flaut, C.: A Clifford algebra associated to generalized Fibonacci quaternions. Adv. Differ. Equ. 2014, 279 (2014). https://doi.org/10.1186/1687-1847-2014-279

12. Flaut, C., Savin, D.: Quaternion algebras and generalized Fibonacci-Lucas quaternions. Adv. Appl. Clifford Algebr. 25, 853-862 (2015). https://doi.org/10.1007/s00006-015-0542-0

13. Flaut, C., Shpakivskyi, V.: On generalized Fibonacci quaternions and Fibonacci-Narayana quaternions. Adv. Appl. Clifford Algebr. 23, 673-688 (2013). https://doi.org/10.1007/s00006-013-0388-2

14. Horadam, A.F.: Basic properties of a certain generalized sequence of numbers. Fibonacci Quart. 3(3), $161-176$ (1965) 
15. Horadam, A.F.: Complex Fibonacci Numbers and Fibonacci Quaternions. American Mathematical Monthly 70, 289-291 (1963). https://doi.org/10.2307/2313129

16. Jafari, M., Yayli, Y.: Generalized quaternions and their algebraic properties. Commun. Fac. Sci. Univ. Ankara Ser. A1 Math. Stat. 64(1), 15-27 (2015)

17. Kösal, H.H., Akyiğit, M., Tosun, M.: Consimilarity of commutative quaternion matrices. Miskolc Math. Notes 16, 965-977 (2015). https://doi.org/10.18514/MMN.2015.1421

18. Kösal, H.H., Tosun, M.: Commutative quaternion matrices. Adv. Appl. Clifford Algebr. 24, 769-779 (2014). https://doi.org/10.1007/s00006-014-0449-1

19. Kösal, H.H., Tosun, M.: Some equivalence relations and results over the commutative quaternions and their matrices. Analele Universitatii "Ovidius" Constanta - Seria Matematica 25(3), 125-142 (2017). https://doi.org/10.1515/auom-2017-0040

20. Kösal, H.H., Tosun, M.: Universal similarity factorization equalities for commutative quaternions and their matrices. Linear Multilinear Algebr. 67, 926-938 (2019). https://doi.org/10.1080/03081087. 2018.1439878

21. Lie, S., Scheffers, M.G.: Vorlesungen über continuerliche Gruppen, Kap. 21. Teubner, Leipzig (1893)

22. Pei, S.-C., Chang, J.-H., Ding, J.-J.: Commutative reduced biquaternions and their Fourier transform for signal and image processing applications. IEEE Trans. Signal Process. 52(7), 2012-2031 (2004). https://doi.org/10.1109/TSP.2004.828901

23. Pogorui, A.A., Rodríguez-Dagnino, R.M., Rodríguez-Said, R.D.: On the set of zeros of bihyperbolic polynomials. Complex Var. Elliptic Equ. 53(7), 685-690 (2008). https://doi.org/10.1080/ 17476930801973014

24. Rochon, D., Shapiro, M.: On algebraic properties of bicomplex and hyperbolic numbers. Anal. Univ. Oradea. Fasc. Math. 11, 71-110 (2004)

25. Scorza, G.: Corpi numerici ed Algebre. (Numerical corps and algebras), Principato, Messina (1921)

26. Segre, C.: Le Rappresentazioni Reali delle Forme Complesse a Gli Enti Iperalgebrici. Mathematische Annalen 40, 413-467 (1892). https://doi.org/10.1007/BF01443559

27. Szynal-Liana, A., Włoch, I.: Hypercomplex numbers of the Fibonacci type. Oficyna Wydawnicza Politechniki Rzeszowskiej, Rzeszów (2019)

Publisher's Note Springer Nature remains neutral with regard to jurisdictional claims in published maps and institutional affiliations. 